\title{
Conventional Pre-operative Chemoradiotherapy and Post-operative Adjuvant Chemotherapy with Induction Chemotherapy Followed by Chemoradiotherapy and Surgery in Locally Advanced Rectal Cancer
}

\author{
Carlos Fernández-Martos, MD, ${ }^{1}$ Rafael Estevan, MD² and Joan Maurel, MD, PhD ${ }^{3}$
}

1. Head, Gastrointestinal Oncology Unit, Medical Oncology Department, Valencia Oncology Institute; 2. Head, Surgery Department, Valencia Oncology Institute;

3. Head, Gastrointestinal Oncology Service, Medical Oncology Department, Hospital Clinic, Barcelona

\begin{abstract}
In locally advanced rectal cancer, pre-operative combined-modality treatment with 5-fluorouracil and radiation has become standard, as it improves locoregional control. However, to date, no improvement has been demonstrated in terms of survival outcomes. Phase III trials published in the past decade included clinically staged populations of heterogeneous risk, and one recurring finding was that there was poor adherence to the adjuvant chemotherapy treatment after surgery. No randomized studies have been able to show that the administration of adjuvant chemotherapy in patients undergoing pre-operative radiochemotherapy improves outcomes, compared to observation. One strategy to address this is to give upfront chemotherapy prior to pre-operative chemoradiotherapy. Compared with post-operative chemotherapy, the upfront strategy does not appear to improve pathological complete response, but it has been found to have a better toxicity profile, improve compliance with treatment, and provide greater exposure to systemic treatment. This can be important for patients at higher risk of distant disease relapse. Introducing a systemically active combination chemotherapy prior to chemoradiotherapy and surgery in an appropriately selected high-risk population may well be the next step in phase III testing in order to improve disease-free survival.
\end{abstract}

\section{Keywords}

Neoadjuvant therapy, locally advanced rectal cancer, induction chemotherapy

Disclosure: The authors have no conflicts of interest to declare.

Received: January 9, 2012 Accepted: January 20, 2012 Citation: Oncology \& Hematology Review, 2012;8(1):48-54 DOI: 10.17925/OHR.2012.08.1.48

Correspondence: Carlos Fernández-Martos, MD, Fundación Instituto Valenciano de Oncología, C/ Beltrán Báguena, 846009 Valencia, Spain. E: cfmartos@fivo.org

In North America and in many European countries, pre-operative 5-fluorouracil (5-FU) and radiation followed by total mesorectal excision (TME) and post-operative adjuvant 5 -FU is one of the standard treatments for locally advanced rectal cancer (LARC) stage II and III. This approach has resulted in a five-year cumulative local relapse rate of less than $10 \%$ and an incidence of distant metastases of about $35 \%{ }^{1,2}$

Phase III trials published in the past decade have included patients with stage I, II, and III and with clinically staged II and III rectal cancer. ${ }^{1-3}$ The most significant conclusions are that pre-operative combined-modality treatment with chemotherapy and long-term chemoradiotherapy has a major impact on local control. However, there has been no impact on disease-free survival (DFS) or overall survival (OS). Since survival is mainly determined by distant metastases, efforts should be directed toward preventing systemic disease.

Recent landmark trials have compared DFS rates following adjuvant therapy in patients with stage II and III colon cancer versus patients with stage II and III rectal cancer, and the results indicate less favorable outcomes in the latter group (see Figure 1). Although the reasons for this are not yet fully understood, the difference may be explained, in part, by the higher incidence of local relapse, the heterogeneous risk population, and the limited and poor efficacy of adjuvant post-operative chemotherapy in patients undergoing pre-operative chemoradiotherapy.

In this article, we will review the main achievements and shortcomings of the standard pre-operative combined-modality treatment followed by surgery and adjuvant chemotherapy, as well as recent data from trials that explored the newer strategy of induction chemotherapy followed by chemoradiation and surgery.

\section{Pre-operative Combined-Modality Treatment, Surgery, and Adjuvant Chemotherapy in Stage II} and III Rectal Cancer

The following first section of the article looks at the achievements and shortcomings of standard pre-operative combined-modality treatment.

\section{Landmark Trials}

Two critical questions have definitively been answered during the past decade: the question of the timing of chemoradiotherapy (before or after surgical resection) and whether or not pre-operative chemoradiotherapy is better than radiotherapy alone. 
Pre-operative versus Post-operative Chemoradiotherapy

Two Phase III trials compared pre-operative with post-operative chemoradiotherapy in clinical stages $\mathrm{T} 3$ or $\mathrm{T} 4$ or $\mathrm{N}+$ rectal cancer.

In the German CAO/ARO/AIO (Chirurgische Arbeitsgemeinschaft Onkologie/Arbeitsgemeinschaft Radioonkologie/Arbeitsgemeinschaft Internistische Onkologie) 94 trial, ${ }^{1}$ patients who received pre-operative therapy had a significantly lower incidence of local recurrence ( $6 \%$ versus $13 \% ; p=0.006$ ), a significantly lower incidence of acute ( $27 \%$ versus $40 \% ; p=0.001$ ) and chronic ( $14 \%$ versus $24 \% ; p=0.012$ ) toxicity, a higher incidence of sphincter preservation (39 \% versus $20 \%$; $p=0.005$ ), and no difference in five-year survival (74 \% versus $76 \%$ ) or five-year cumulative distant recurrence rates (36 \% versus $38 \%$ ). Updated data after a median follow-up of 11 years showed a persisting significant improvement of local control following pre-operative versus post-operative chemoradiotherapy. However, there was no effect on OS. ${ }^{4}$

The NSABP (National Surgical Adjuvant Breast and Bowel Project) R-03 trial $^{5}$ was the first to document a significant improvement in DFS (65 \% versus $53 \% ; p=0.011)$ in patients undergoing neoadjuvant chemoradiotherapy compared with post-operative chemoradiotherapy. There was no difference in five-year local recurrence between the neoadjuvant chemoradiotherapy arm and the post-operative chemoradiotherapy arm (10.7 \% for each). Patients in the neoadjuvant chemoradiotherapy arm received six weeks of bolus 5-FU and leucovorin (cycle 1) before the start of radiotherapy. Two courses of chemotherapy (cycles 2 and 3) were administered during the first and fifth week of radiotherapy and four courses (cycles 4-7) were administered post-operatively. However, in the NSABP R-03 trial, ${ }^{5}$ only 267 patients were recruited instead of the 900 patients the investigators had originally planned to enroll, which limited the trial's statistical power to detect differences. In contrast, the CAO/ARO/AIO 94 trial $^{1}$ succeeded in randomly assigning more than 800 patients to treatment, with longterm follow-up data confirming the results, and it therefore remains the definitive trial of chemoradiotherapy in rectal cancer. ${ }^{\circ}$

\section{Pre-operative Radiotherapy with or without Concurrent Chemotherapy}

The second question is whether or not conventional pre-operative chemoradiotherapy is better than radiotherapy alone in LARC. Three recent Phase III trials have tackled this issue.

The French FFCD 9203 trial $^{3}$ (ClinicalTrials.gov identifier NCT00296608) randomized resectable stage T3/T4 patients with rectal adenocarcinoma to receive pre-operative radiotherapy (45 Gy) with or without concurrent bolus 5-FU/leucovorin. Surgery was planned three to 10 weeks after the end of radiotherapy. All patients were to receive the same adjuvant chemotherapy regimen of 5-FU/leucovorin. Pathological complete response $(\mathrm{pCR})$ was more frequent with chemoradiotherapy $(11.4 \%$ versus $3.6 \%$; $p<0.05)$. The five-year incidence of local recurrence was lower with chemoradiotherapy $(8.1 \%$ versus $16.5 \%$; $p<0.05)$. However, there was no difference between the two arms in five-year progression-free survival (PFS) or OS.

The randomized EORTC 22921 trial $^{2}$ (ClinicalTrials.gov identifier NCT00002523) evaluated the role of concurrent chemoradiotherapy in
Figure 1: Five-year Disease-Free Survival in Landmark Clinical Trials in Colon and Rectal Cancer

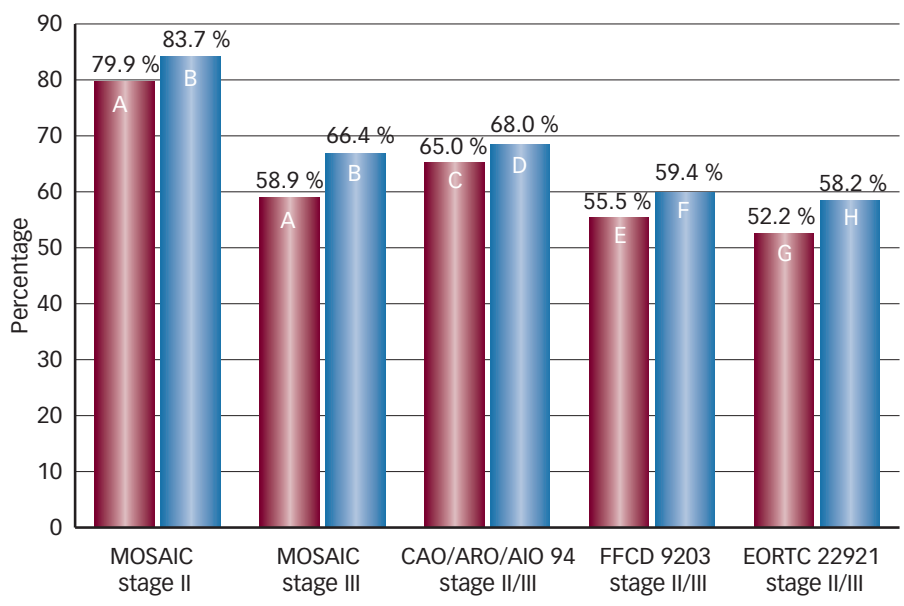

Trial in colon cancer: MOSAIC (André et al., 2004'12); trials in rectal cancer: CAO/ARO/AIO 94 (Sauer et al., 2004'), FFCD 9203 (Gérard et al., 20063), and EORTC 22921 (Bosset et al., 2006'). A: 5-FU/LV; B: FOLFOX; C: Pre-operative 5-FU/RT, post-operative 5-FU; D: Post-operative 5-FU/RT 5-FU; E: Pre-operative RT, post-operative 5-FU; F: Pre-operative 5-FU/RT, post-operative 5-FU; G: Pre-operative 5-FU/RT or RT, no post-operative FU; H: Pre-operative FU/RT or RT, post-operative FU.

5-FU = 5-fluorouracil; FOLFOX = leucovorin, fluorouracil, and oxaliplatin; LV = leucovorin; $R T=$ radiotherapy.

the neoadjuvant setting, along with the role of adjuvant chemotherapy in patients with resectable stage T3/T4 rectal cancer. Patients $(n=1,011)$ were randomized to one of four treatment arms:

- pre-operative radiotherapy (45 Gy over five weeks) alone;

- pre-operative radiotherapy plus two five-day chemotherapy courses with bolus 5-FU and leucovorin in week 1 and 5 of radiotherapy;

- pre-operative radiotherapy plus four post-operative courses of chemotherapy (bolus 5-FU/leucovorin); or

- pre-operative and chemotherapy plus post-operative chemotherapy.

The five-year cumulative incidence rates for local recurrences were $8.7 \%, 9.6 \%$, and $7.6 \%$ in the groups who received chemotherapy pre-operatively, post-operatively, or both, respectively, and $17.1 \%$ in the group that did not receive chemotherapy $(p=0.002)$. No differences were observed between the four arms in distant disease relapse. Overall, the five-year cumulative incidence of distant metastases was $34.4 \%$. The authors therefore concluded that, regardless of timing, chemotherapy provides a significant benefit with respect to local control. No beneficial effects were seen in PFS and OS in the chemoradiotherapy groups versus the radiotherapy group.

The third trial ${ }^{7}$ included 207 patients with locally non-resectable T4 rectal cancer or local recurrence who received either pre-operative chemotherapy (5-FU/leucovorin) administered concurrently with radiotherapy (50 Gy) plus adjuvant chemotherapy for 16 weeks after surgery, or pre-operative radiotherapy alone (50 Gy). Local control (82 \% versus $67 \%$ at five years; $p=0.03$ ) and cancer-specific survival ( $72 \%$ versus $55 \% ; p=0.02$ ) rates were better in the chemoradiotherapy group. However, once again, there were no differences in OS. It should be noted that, in the three trials described above, acute grade 2 or higher toxicity (mainly gastrointestinal) was significantly more common, although 
Table 1: Phase III Trials of Pre-operative Fluoropirymidine/Radiation versus Fluoropirymidine/Oxiplatin/Radiation

\begin{tabular}{|c|c|c|c|c|c|c|c|}
\hline Trial & $n$ & Pre-operative Treatment & Primary Endpoint & \% урт0 N0 p & p-value & $\% \mathrm{~N}+$ & $\%$ RO \\
\hline $\mathrm{ACCORD}^{18}$ & 598 & $\mathrm{RT}+\mathrm{CAP}$ & Pathologic & 13.9 & & 30.5 & 87 \\
\hline (NCT00227747) & & RT + CAPOX & complete response & 19.2 & $p=0.09$ & 28.5 & 92 \\
\hline \multirow[t]{2}{*}{ STAR- $01^{19}$} & 747 & $\mathrm{RT}+5-\mathrm{FU}$ & Overall survival & 16 & & 26.0 & 94 \\
\hline & & $\mathrm{RT}+\mathrm{FOLFOX}$ & & 16 & $p=0.9$ & 29.0 & 97 \\
\hline NSABP R-0 $4^{17}$ & 1,608 & RT-5-FU/CAP & 3-year local-regional & 19.1 & & 29.0 & - \\
\hline (NCT00058474) & & RT-FOLFOX/CAPOX & relapse rate & 20.9 & $p=0.46$ & 29.6 & - \\
\hline $\mathrm{CAO} / \mathrm{ARO} / \mathrm{AIO} 04^{20}$ & 1,265 & RT-5-FU & 3-year disease-free & 12.8 & & 30.0 & 92 \\
\hline (NCT00349076) & & RT-FOLFOX & survival & 16.5 & $p=0.045$ & 28.0 & 90 \\
\hline
\end{tabular}

5-FU = 5-fluorouracil; CAP = capecitabine; CAPOX = capecitabine plus oxaliplatin; FOLFOX = leucovorin, fluorouracil, and oxaliplatin; $N+=$ node positivity; $R T=$ radiotherapy.

Table 2: Phase II Studies of Induction Chemotherapy Followed by Chemoradiotherapy with or without Adjuvant Chemotherapy in Rectal Cancer Patients

\begin{tabular}{|c|c|c|c|c|c|}
\hline Study & $n$ & Inclusion Criteria & Treatment & Toxicity & pCR \\
\hline Chau et al., $2003^{22}$ & 36 & $\begin{array}{l}\text { MRI or CT + DRE T3/T4 } \\
\mathrm{NX} /+ \\
\text { M0 }\end{array}$ & $\begin{array}{l}\text { - Induction CT: PVI 5-FU+MMC } 12 \text { weeks } \\
\text { - CRT: PVI 5-FU during RT (50.4-54.0 Gy) } \\
\text { - Surgery: 4-6 weeks after CRT } \\
\text { - Adjuvant CT: PVI 5-FU, MMC } 12 \text { weeks }\end{array}$ & $\begin{array}{l}\text { - Induction CT: G3/4 } 25 \text { \% } \\
\text { - CRT: G3/4 } 28 \text { \% (skin) } \\
\text { - Surgery: } 1 \text { death (anastomotic leak } \\
\text { with multi-organ failure) }\end{array}$ & $2.7 \%$ \\
\hline $\begin{array}{l}\text { Chau et al., } 2006 \\
\text { (updated Chua et al., } \\
2010)^{23,24}\end{array}$ & 105 & $\begin{array}{l}\text { MRI: T3 } \leq 1 \mathrm{~mm} \text { to CRM, } \\
\text { T3 at or below levators; } \\
\text { T3 } \geq 5 \text { mm into fat; } \\
\text { T4; N2; M0 }\end{array}$ & $\begin{array}{l}\text { - Induction CT: CAPOX } 4 \text { cycles } \\
\text { - CRT: CAP during RT (54 Gy) } \\
\text { starting at week } 13 \\
\text { - Surgery: } 6 \text { weeks after CRT; } \\
\text { - Adjuvant CT: CAP } 4 \text { cycles }\end{array}$ & $\begin{array}{l}\text { - Induction CT: } 5 \text { deaths, } 1 \text { neutropenic } \\
\text { sepsis, } 2 \text { pulmonary embolism, } \\
1 \text { myocardial infarction, } 1 \text { cardiac failure } \\
\text { - G3-5 diarrhea } 10 \% \\
\text { - CRT: G3-5 } 42 \% \text { (skin) } \\
\text { - surgery: no deaths within } 30 \text { days }\end{array}$ & $20 \%$ \\
\hline Calvo et al., $2006^{25}$ & 52 & $\begin{array}{l}\text { US T3/T4 or } \mathrm{N}+ \\
\text { M0 }\end{array}$ & $\begin{array}{l}\text { - Induction CT: FOLFOX4 } 2 \text { cycles } \\
\text { - CRT: tegafur during RT (45.0-50.4 Gy) } \\
\text { starting on day } 30 \\
\text { - Surgery: 4-6 weeks after CRT plus } \\
\text { IORT boost ( } 10-15 \mathrm{~Gy} \text { ) } \\
\text { - Adjuvant CT: left to the treating } \\
\text { oncologist's discretion }\end{array}$ & $\begin{array}{l}\text { - Induction CT: G3 } 6 \text { \% } \\
\text { - CRT: G3-4 } 33 \% \\
\text { - Surgery: } 31 \% \text { post-operative } \\
\text { complications }\end{array}$ & $\begin{array}{l}\text { NR } \\
\text { ypT0: } 29 \%\end{array}$ \\
\hline Koeberle et al., $2008^{26}$ & 60 & $\begin{array}{l}\text { MRI or US T3/T4 NX/+ } \\
\text { M0 }\end{array}$ & $\begin{array}{l}\text { - Induction CT: CAPOX } 1 \text { cycle } \\
\text { - CRT: CAP and OXA during RT (45 Gy) } \\
\text { starting on day } 22 \\
\text { - Surgery: } 5-6 \text { weeks after CRT } \\
\text { - Adjuvant CT: left to the treating } \\
\text { oncologist's discretion }\end{array}$ & $\begin{array}{l}\text { - Induction CT: G3 } 17 \% \text { diarrhea, } \\
\text { G4 } 3 \text { \% infection, } 1 / 60 \text { death } 19 \text { days } \\
\text { after start due to neutropenic sepsis } \\
\text { - CRT: G3 } 17 \% \text { diarrhea } \\
\text { - Surgery: NR }\end{array}$ & $23 \%$ \\
\hline $\begin{array}{l}\text { Gunnlaugsson et al., } \\
2009^{27}\end{array}$ & 49 & $\begin{array}{l}\text { 'Non-resectable' } \\
\text { colorectal carcinoma } \\
\text { T4NxM0-1; } \\
41 \text { points rectal }\end{array}$ & $\begin{array}{l}\text { - Induction CT: CAPOX } 2 \text { cycles } \\
\text { - CRT: CAP and OXA during RT (50.4 Gy) } \\
\text { starting at week } 7 \\
\text { - surgery: performed if feasible }\end{array}$ & $\begin{array}{l}\text { - Induction CT: G3/4 } 11 \% \text { diarrhea } \\
\text { - CRT: G3/4 } 24 \text { \% diarrhea } \\
\text { - } 1 \text { death due to myocardial infarction }\end{array}$ & $13 \%$ \\
\hline
\end{tabular}

5-FU = 5-fluorouracil; $C A P=$ capecitabine; $C A P O X=$ capecitabine plus oxaliplatin; $C R M=$ circumferential resection margin; $C R T=$ chemoradiotherapy; $C T=$ chemotherapy; DRE = digital rectal examination; FOLFOX = leucovorin, fluorouracil, and oxaliplatin; IORT = intra-operative radiotherapy; $M M C=$ mitomycin $C ;$; $M R I=$ magnetic resonance imaging; $N+=$ node positivity; NR = not reported; OXA = oxaliplatin; $P C R=$ pathological complete response; $P V I=$ portal vein infusion; $R T=$ radiotherapy; US = ultrasonography. Adapted from Rödel et al., $2010 .{ }^{31}$

staying within safe limits, in patients assigned to pre-operative chemoradiotherapy. No differences were noted in late toxicity.

\section{Adjuvant Therapy After Chemoradiotherapy and Surgery}

The benefit of adjuvant therapy after pre-operative chemoradiotherapy has not been well established in randomized trials. The most relevant published trial was the aforementioned EORTC 22921 trial, in which, after a median follow-up of 5.4 years, adjuvant chemotherapy did not appear to confer any benefits in terms of DFS or OS. There are, however, some important points to note. The first is that, in the EORTC 22921 trial, $26.9 \%$ of patients randomized to receive adjuvant chemotherapy never actually started the treatment and only $43 \%$ received $95 \%$ of the planned dose of chemotherapy. Data from other recent Phase II and Phase III trials ${ }^{1,8-10}$ are similar, showing that applying chemotherapy (5-FU or CAPOX [capecitabine plus oxaliplatin]) after pre-operative chemoradiation and surgery is problematic and often not feasible at adequate doses. More recently, a National Comprehensive Cancer Network (NCCN) analysis carried out on 810 stage II and III patients from eight specialised cancer centers showed that $20 \%$ of those treated with neoadjuvant chemoradiation did not receive any adjuvant chemotherapy, the most common reasons being: patient decision to abandon treatment because of fatigue following the aggressive therapy 
schedule; toxicity; and physician recommendation due to comorbid illness. ${ }^{11}$ By contrast, adherence to the planned treatment is higher in colon cancer trials. In the MOSAIC trial ${ }^{12}$ (ClinicalTrials.gov identifier NCT00275210), $98.6 \%$ of patients received at least one cycle of FOLFOX (leucovorin, fluorouracil, and oxaliplatin) and $98.9 \%$ of patients received at least one cycle of 5-FU/leucovorin; $74.7 \%$ of patients in the FOLFOX group and $86.5 \%$ of patients in the 5 -FU/leucovorin group received the planned 12 cycles.

The second point is that the EORTC 22921 trial included clinically staged T3/T4 patients, ${ }^{13} 35 \%$ of whom had been recruited after a clinical examination that used some subjective criteria (e.g., a clinically staged T3 patient had to have one of the following at digital rectal examination: a circular extension, a lateral or posterior fixity, or an anterior adhesion to the prostate or invasion of the recto-vaginal wall); $60 \%$ were ultrasound-staged T3 and, as the German study showed, ${ }^{1}$ about $20 \%$ may have been stage I. Therefore, in this trial, the true population may have been made of stages I, II, and III patients.

Third, the survival curves of patients who received post-operative adjuvant chemotherapy begin to diverge after two years for DFS and after four years for OS from those of patients who did not receive adjuvant chemotherapy, suggesting that further follow-up is required to determine whether these divergences will continue.

An updated analysis was subsequently performed to determine the long-term results of the EORTC 22921 trial. ${ }^{14}$ The goal of this exploratory multivariate analysis was to investigate whether it would be possible to identify a subgroup of patients who would benefit most from adjuvant chemotherapy in the long term. Adjuvant chemotherapy did not improve DFS or OS in all patients with resectable T3/T4 rectal cancer. However, a subset analysis revealed that patients whose disease responded to neoadjuvant therapy-specially those whose tumors were downstaged from урт3-4 to yрт0-2 with pre-operative therapy-did experience survival benefits from adjuvant chemotherapy.

More recently, a large retrospective analysis explored the same issue; 2,724 patients were included, $41 \%$ of whom underwent adjuvant chemotherapy (mostly 5 -FU-based). ${ }^{15}$ The aim was to determine whether patients with pCR, patients with ypT1-2 tumor, and patients with ypT3-4 tumor after chemoradiotherapy benefited differently from adjuvant chemotherapy in terms of DFS. The hazard ratio for DFS following adjuvant chemotherapy was 0.94 (95\% confidence interval [Cl] 0.50-1.78) in patients with pCR, 0.61 (95\% Cl 0.40-0.92) in patients with ypT1-2 tumors, and 0.97 (95\% Cl 0.75-1.25) in patients with ypT3-4 tumors. Patients with ypT01-2 NO benefited most and pN-stage did not alter the benefits of adjuvant chemotherapy for ypT3-4 patients. ${ }^{15}$

\section{Pre-operative Chemoradiotherapy-Novel Regimens} Continuous-infusion 5-Fluorouracil versus Capecitabine Combined with Radiotherapy

Although it has never been formally compared with bolus 5-FU in the neoadjuvant setting in rectal cancer, continuous-infusion 5-FU (225 mg/m² five days per week) concomitant with 45.0-50.4 Gy
Figure 2: Schematic View of the Randomized Phase II Grupo Cáncer de Recto 3 Trial

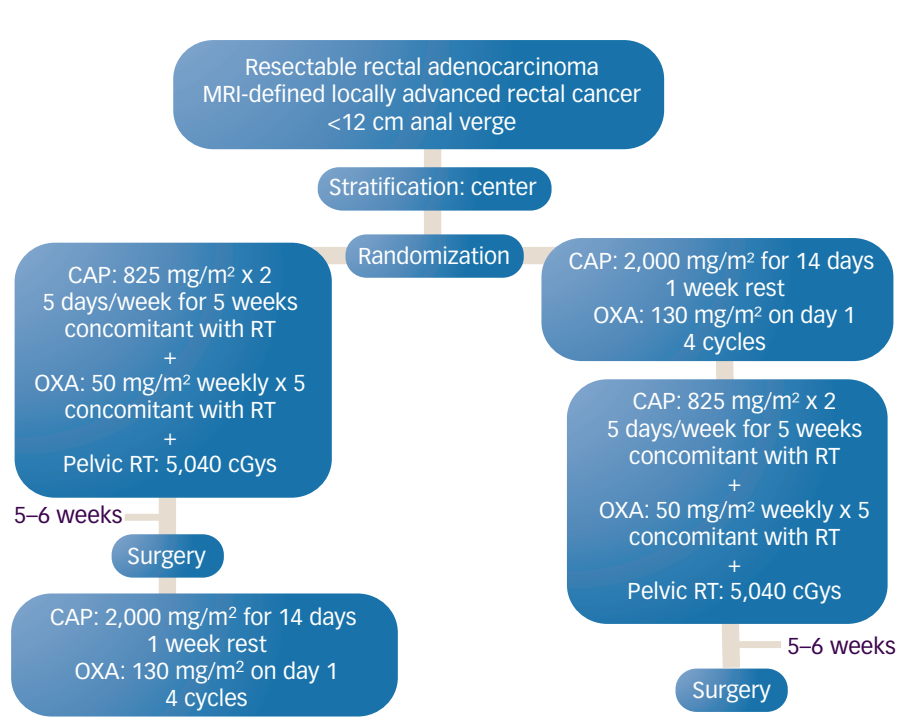

$C A P=$ capecitabine; $M R I=$ magnetic resonance imaging; $O X A=$ oxaliplatin $; T=$ radiotherapy . Adapted from Fernandez-Martos et al., 2010. ${ }^{28}$

Table 3: Results of the Randomized Phase II Grupo Cáncer de Recto 3 Trial

\begin{tabular}{|c|c|c|c|}
\hline & $\begin{array}{l}\text { CRT + TME } \\
\text { Adjuvant } \\
\text { CAPOX } \\
(n=56)\end{array}$ & $\begin{array}{l}\text { Induction } \\
\text { CAPOX + } \\
\text { CRT + TME } \\
(n=52)\end{array}$ & $p$-value \\
\hline \multicolumn{4}{|l|}{ Efficacy } \\
\hline RO resection rates & $87 \%$ & $86 \%$ & NS \\
\hline $\mathrm{pCR}$ & $13 \%$ & $14 \%$ & NS \\
\hline \multicolumn{4}{|l|}{ Compliance } \\
\hline CAPOX $\times 4$ & $57 \%$ & $94 \%$ & 0.0001 \\
\hline \multicolumn{4}{|l|}{ Toxicity G3-4 } \\
\hline CAPOX-RT & $29 \%$ & $23 \%$ & 0.36 \\
\hline CAPOX & $54 \%$ & $19 \%$ & 0.0004 \\
\hline
\end{tabular}

CAPOX = capecitabine plus oxaliplatin; $C R T=$ chemoradiotherapy; NS = not supplied; $P C R=$ pathological complete response; $R T=$ radiotherapy; $T M E=$ total mesorectal excision Adapted from Fernandez-Martos et al., 2010. ${ }^{28}$

radiotherapy is currently considered the standard of care in the US and many European countries in stages II and III patients.

Results of two large head-to-head comparisons between 5-FU and capecitabine in the pre-operative setting were reported in abstract form in 2011. 16,17 The German MARGIT (Mannheimer Arbeitsgruppe für Gastrointestinale Tumoren) trial ${ }^{16}$ started in 2001 comparing 5-FU versus capecitabine as part of the post-operative treatment of stage II-III rectal cancer. When the German CAO/ARO/AIO 94 trial was published, a neoadjuvant stratum was initiated. Patients $(n=161)$ were randomly assigned to receive neoadjuvant therapy with capecitabine $\left(1,650 \mathrm{mg} / \mathrm{m}^{2}\right.$ on days $\left.1-38\right)$ plus radiation (50.4 Gy) or 5-FU (225 mg/m² on days $1-38$ ) plus radiation (50.4 Gy). In this neoadjuvant stratum, patients treated with capecitabine plus radiation exhibited a higher, although non-significant, rate of T-downstaging (defined as ypT0-2; 52 \% 
versus $39 \% ; p=0.16$ ) and a higher rate of $p C R$ (13.5 \% versus $5.4 \%$; $p=0.16)$. Adding oxaliplatin to either strategy did not improve outcomes but did increase toxicity, especially grade 3 or 4 diarrhea. With a median follow-up of 52 months, in the overall population of the MARGIT trial $(n=392)$, there was a significant improvement in DFS and non-inferiority in OS for capecitabine versus 5-FU as part of adjuvant or neoadjuvant chemoradiotherapy. The side-effect profiles of the two drugs differed, with a greater incidence of hand-foot syndrome, proctitis, diarrhea, and fatigue with capecitabine, and more alopecia and leukopenia with 5-FU.

The NSABP R-04 trial ${ }^{17}$ (ClinicalTrials.gov identifier NCT00058474) compared the efficacy of four chemotherapy regimens administered concomitantly with pre-operative radiotherapy; 1,608 patients with clinical stage II or III rectal cancer undergoing pre-operative radiotherapy (4,500 cGy in 25 fractions over five weeks plus a boost of 540-1,080 cGy in three-to-six daily fractions) were randomly assigned to one of the following regimens: continuous-infusion intravenous (IV) 5 -FU (225 mg/m² five days per week), with or without IV oxaliplatin (50 mg/m² per week x 5); oral capecitabine ( $825 \mathrm{mg} / \mathrm{m}^{2}$ twice daily five days per week), with or without oxaliplatin ( $50 \mathrm{mg} / \mathrm{m}^{2}$ per week $x$ 5). There were no differences in the rate of grade $3 / 4$ diarrhea between capecitabine- and 5-FU-treated patients, although adding oxaliplatin to either strategy did increase toxicity, especially grade 3/4 diarrhea (6.6\% versus $15.4 \% ; p=0.0001)$. Secondary endpoints showed similar rates between the capecitabine and 5-FU regimens: surgical downstaging $23 \%$ versus $20.7 \%(p=0.62)$, sphincter-saving surgery $62.7 \%$ versus $61.2 \%(p=0.59)$, and $\mathrm{pCR} 22.2 \%$ versus $18.8 \%$ ( $p=0.12)$. No significant differences between the two arms were found for node positivity $(\mathrm{N}+)$ or surgical complications. The definitive analysis of local tumor control (the primary endpoint), DFS, and OS will be performed in 2013.

Overall, these results support the equivalence in both efficacy and safety of capecitabine and IV infusional 5-FU in the pre-operative chemoradiotherapy management of rectal cancer patients.

\section{Fluoropyrimidines versus Fluoropyrimidines with Oxaliplatin Concomitant with Radiotherapy}

One approach to improve outcomes in rectal cancer is to deliver a second radiation-sensitizing drug with effective systemic activity. The French ACCORD12/0405-Prodige 2 trial $^{18}$ (ClinicalTrials.gov identifier NCT00227747) compared neoadjuvant radiotherapy 45 Gy/25 fractions plus concurrent capecitabine $800 \mathrm{mg} / \mathrm{m}^{2}$ twice daily five days per week, with radiotherapy 50 Gy/25 fractions plus capecitabine $800 \mathrm{mg} / \mathrm{m}^{2}$ twice daily five days per week plus oxaliplatin $50 \mathrm{mg} / \mathrm{m}^{2}$ once weekly for five weeks. It found no differences in terms of PCR (14\% versus $19 \%$; $\mathrm{p}=0.09$ ) or sphincter-sparing surgery (75 \% versus $77 \%$ ) rates. In addition, the overall rate of grade 3-4 toxicity was $10.9 \%$ with radiotherapy plus capecitabine versus $25.4 \%$ with radiotherapy plus CAPOX $(p=0.001)$.

These results are in concordance with the Italian Phase III STAR-01 trial $^{19}$ comparing infusional 5-FU plus weekly oxaliplatin plus radiotherapy with infusional 5-FU plus radiotherapy. In this trial, the addition of weekly oxaliplatin to standard 5-FU-based pre-operative chemoradiotherapy significantly increased toxicity without any effect on local tumor response.
Moreover, the aforementioned NSABP R-04 randomized trial ${ }^{17}$ showed that adding weekly oxaliplatin to infusional 5-FU or capecitabine did not improve outcomes but added significant toxicity.

Recently, the German CAO/ARO/AIO-04 ${ }^{20}$ (ClinicalTrials.gov identifier NCT00349076) reported some early preliminary results. This trial randomized patients $(n=1,265)$ to receive either pre-operative chemoradiotherapy (50.4 Gy) plus 5-FU or chemoradiotherapy plus 5-FU plus oxaliplatin. In contrast with the trials mentioned above, a significant increase in pCR rates (12.8\% in the 5-FU arm and $16.5 \%$ in the 5 -FU plus oxaliplatin arm; $\mathrm{p}=0.045)$ with no increase in toxicity was observed. It should be emphasized that the 5-FU scheme used in the control arm (5-FU $1,000 \mathrm{mg} / \mathrm{m}^{2}$ on days $1-5$ and 29-33) was different from that used in the 5-FU plus oxaliplatin arm (5-FU $250 \mathrm{mg} / \mathrm{m}^{2}$ on days 1-14 and 22-35) and oxaliplatin was administrated with a week off, thus allowing greater compliance with the treatment. No differences were found in other efficacy endpoints (i.e., RO resection and good TME surgery).

In summary, up to now, the impact of neoadjuvant oxaliplatin in rectal cancer-if any-has been small. The CAO/ARO/AIO-04 trial was the only one to show a small but significant difference in $\mathrm{PCR}$ rates in patients who had oxaliplatin added to their treatment. Other secondary efficacy endpoints were similar in all the trials mentioned above (summarized in Table 1). As the follow-up has been short, no data regarding primary endpoints are currently available.

\section{Induction Chemotherapy Followed by Concomitant Chemoradiotherapy and Surgery}

Treatment of high-risk patients with fluoropyrimidines and concurrent radiotherapy without the addition of adequate doses and cycles of chemotherapy appears to be less than optimal. Strategies are needed to improve adherence to the systemic component of the treatment.

One approach to address this issue is to deliver induction chemotherapy before pre-operative chemoradiotherapy. Induction chemotherapy may be associated with better treatment compliance and may allow full systemic doses of chemotherapy to be delivered. Other theoretical advantages include the possibility of shrinking or downstaging a locally advanced tumor, thereby facilitating more effective local treatment and early treatment of micrometastases. Disadvantages include the delay in surgery and the reduced efficacy of subsequent radiotherapy with selection of radiotherapy-resistant clones. ${ }^{21}$

\section{Phase II Studies}

The first published trial of such a strategy is a study from the UK ${ }^{22}$ in which $36 \mathrm{~T} 3 / \mathrm{T} 4$ and NX/+ rectal cancer patients were given 12 weeks of induction protracted venous infusion 5-FU and mitomycin $\mathrm{C}$ before standard chemoradiation and surgery. A high rate of tumor downstaging was recorded, with no disease progression during neoadjuvant chemotherapy, and $82 \%$ of patients achieved microscopically clear R0 resections despite nearly one-third having had tumors that were initially threatening or involving the circumferential resection margin (CRM). However, only one pCR was achieved.

More recently, investigators from the same group published the largest Phase II trial to date (first published in 2006 with 77 patients, updated in 
2010 with 105 patients and a median follow-up of 55 months), which examined the use of four cycles of induction, this time with CAPOX followed by chemoradiotherapy plus capecitabine. ${ }^{23,24}$ This trial included high-risk rectal cancer patients as defined by magnetic resonance imaging (MRI). Eligible patients had to have tumors within $1 \mathrm{~mm}$ of mesorectal fascia (i.e., CRM threatened), T3 tumors at or below levators, tumors extending $5 \mathrm{~mm}$ into perirectal fat, T4 tumors, or T1-4N2 tumors. There was a clinically significant occurrence of cardiac/thromboembolic toxicity during neoadjuvant CAPOX which led to three deaths. This prompted a protocol amendment to exclude the entry of patients with a previous history of stable angina, arrhythmia, acute coronary syndrome (even if controlled by drugs), or myocardial infarction within the previous 12 months. After amendment of the protocol, only one further fatal pulmonary embolism occurred.

High radiological response rates to pre-operative treatment (96\% of assessable patients), $20 \%$ rates of $\mathrm{PCR}$, and no disease progression during pre-operative treatment were recorded. Three-year and five-year failure-free survival rates (intention to treat [ITT] population) were $68 \%$ and $64 \%$, respectively. Three-year and five-year os rates were $83 \%$ and $75 \%$, respectively. Clinical results from this and other phase II trials are summarized in Table $2 .{ }^{22-27}$

\section{Data from Randomized Trials}

On the basis of these encouraging results, our study group (Grupo Cáncer de Recto) designed a phase II randomized trial to compare this approach with conventional pre-operative chemoradiotherapy followed by surgery and post-operative adjuvant chemotherapy (see Figure 2). ${ }^{28}$ A total of 108 patients with LARC defined by MRI of the pelvis were randomly assigned to either pre-operative chemoradiotherapy with CAPOX and concurrent radiation followed by surgery and four cycles of post-operative adjuvant CAPOX, or induction CAPOX followed by chemoradiotherapy and surgery. MRI criteria for defining LARC were as follows: tumors extending $2 \mathrm{~mm}$ within or beyond the mesorectal fascia (i.e., an involved or threatened CRM); lower third ( $\leq 6 \mathrm{~cm}$ from the anal verge) сT3 tumors; resectable cT4 tumors; or any CT3N+ tumors. The primary endpoint was the PCR rate. On an ITT basis, the PCR rate was $13.5 \%$ (95 \% Cl 5.6-25.8 \%) with the standard strategy (adjuvant chemotherapy) and $14.3 \%$ (95 \% Cl 6.4-26.2\%) with the induction strategy. There were no statistically significant differences in other endpoints, including downstaging, tumor regression, and Ro resection.

The most compelling results from this study concern the secondary endpoints. During the course of chemoradiotherapy, there were no differences between the study arms in the number of patients with grade 3-4 toxicity. However, during the course of chemotherapy, significantly more patients had grade 3-4 toxicity in the adjuvant arm than in the induction arm (54\% versus $19 \%$, respectively; $\mathrm{p}=0.0004$ ). Furthermore, $25 \%$ of patients in the adjuvant arm did not begin treatment and only $57 \%$ received all four cycles, whereas $100 \%$ of patients in the induction arm began treatment and $94 \%$ received all four cycles (see Table 3). These differences reached statistical significance.
The updated results with a median follow-up time of 39.3 months showed 36-month DFS rates to be $68 \%$ (95\% Cl 53-80) in the adjuvant arm and $70 \%$ (95 \% Cl 55-80) in the induction arm ( $\mathrm{p}=0.97) .{ }^{29}$ Analyzing the subset of patients at higher risk (i.e., patients with CT4, cT3 distal third, or CRM+ tumors), the 36-month DFS was $62 \%$ in the adjuvant arm versus $70 \%$ in the induction arm. Although the numbers are small, this non-significant difference may indicate a trend toward some benefit from the induction strategy in the higher-risk population.

Recently published preliminary results of a similar trial ${ }^{30}$ show that 57 patients with T2-T4N+ disease were randomized to concomitant chemoradiotherapy with continuous-infusion 5-FU and surgery (arm A) or induction FOLFOX6 followed by the same chemoradiotherapy regimen (arm B). Both pCR rates (27\% in arm A, $25 \%$ in arm B) and tumor regression grades were similar. Induction chemotherapy did not compromise the completion of chemoradiotherapy.

Further evidence that induction chemotherapy can contribute to better control of distant disease relapse comes from the aforementioned NSABP R-03 phase III trial. ${ }^{5}$ This was the first trial to document a significant improvement in DFS in patients undergoing neoadjuvant chemoradiotherapy. Perhaps a six-week course of induction chemotherapy and a greater total cumulative dose of 5-FU are more effective in controlling micrometastatic disease and improving DFS?

\section{Conclusions}

Several conclusions can be drawn from the studies discussed in this article. In LARC, pre-operative combined-modality treatment with 5-FU and radiation, compared with radiotherapy alone, increases the PCR rate and improves locoregional control, but has not so far been demonstrated to improve survival outcomes. The Phase III studies published in the past decade included clinically staged populations of heterogeneous risk, and one of the recurring findings is that there was poor adherence to the adjuvant treatment with chemotherapy after surgery. No randomized studies have been able to show that the administration of adjuvant chemotherapy in patients undergoing pre-operative chemoradiotherapy improves outcomes, compared to observation. Emerging evidence from several phase ॥ trials and, recently, randomized phase $\|$ trials, indicate that induction chemotherapy for rectal cancer is feasible, does not compromise pCR or Ro resection rates, is less toxic, and enables chemotherapy to be delivered at adequate doses and intensity.

Nonetheless, while these data are statistically-and potentially clinically_meaningful, these remarkable findings are regarded as hypothesis-generating. Given the fact that the predominant site of failure after combined-modality treatment is systemic rather than local, we believe that introducing a systemically active combination chemotherapy prior to chemoradiotherapy and surgery in a high-risk population selected by MRI may well be the next step in phase III testing in order to improve DFS.
1. Sauer R, Becker $\mathrm{H}$, Hohenberger W, et al., Preoperative versus postoperative chemoradiotherapy for rectal cancer, N Engl J Med, 2004;351:1731-40.

2. Bosset JF, Collette L, Calais G, et al., EORTC Radiotherapy
Group Trial 22921, Chemotherapy with preoperative

radiotherapy in rectal cancer, N Eng/ J Med, 2006;355:1114-238.

3. Gérard JP, Conroy T, Bonnetain F, et al., Preoperative radiotherapy with or without concurrent fluorouracil and leucovorin in T3-4 rectal cancers: results of FFCD 9203, J Clin Oncol, 2006;24:4620-5.

4. Sauer R, Liersch T, Merkel S, et al., Preoperative versus postoperative chemoradiotherapy for locally advanced rectal 
cancer: results of the German CAO/ARO/AIO-94 randomized phase III trial after a median follow-up of 11 years, I Clin Oncol 2011;29(Suppl.): Abstract 3516.

5. Roh MS, Colangelo LH, O'Connell MJ, et al., Preoperative multimodality therapy improves disease-free survival in patients with carcinoma of the rectum: NSABP R-03, I Clin Oncol, 2009:27:5124-30.

6. Minsky B, Is preoperative chemoradiotherapy still the treatment of choice for rectal cancer? I Clin Oncol, 2009;27:5115-6.

7. Braendengen M, Tveit KM, Berglund A, et al., Randomized phase III study comparing preoperative radiotherapy with chemoradiotherapy in nonresectable rectal cancer, I Clin Oncol, 2008;26:3687-94.

8. Fernandez-Martos C, Aparicio J, Bosch C, et al., Preoperative uracil, tegafur, and concomitant radiotherapy in operable rectal cancer: a phase II multicenter study with 3 years' follow-up, J Clin Oncol, 2004;22:3016-22.

9. Rödel C, Liersch T, Hermann R, et al., Multicenter phase II trial of chemoradiation with oxaliplatin for rectal cancer, I Clin Oncol, 2007; 25:668-74.

10. Sebag-Montefiore $D$, Rutten $H$, Rullier $E$, et al., Three-year survival results of CORE (capecitabine, oxaliplatin, radiotherapy, and excision) study alter postoperative chemotherapy in patients with locally advanced rectal adenocarcinoma, Presented at: American Society of Clinical Oncology 2009 Gastrointestinal Cancers Symposium, San Francisco, January 15-17, 2009; Abstract 447.

11. Khrizman $P$, Niland $J C$, Veer $A$, et al., Postoperative adjuvant chemotherapy (CTX) use in patients (Pts) with stage II/III rectal cancer treated with neoadjuvant therapy: a National Comprehensive Cancer Network (NCCN) analysis, I Clin Oncol, 2011;29(Suppl.):Abstract 3515.

12. André $\mathrm{T}$, Boni $\mathrm{C}$, Mounedji-Boudiaf $\mathrm{L}$, et al., Oxaliplatin, fluorouracil, and leucovorin as adjuvant treatment for colon cancer, N Eng/ J Med, 2004;350:2343-51.

13. Bosset JF, Calais J, Mineur L, et al., Enhanced tumorocidal effect of chemotherapy with preoperative radiotherapy for rectal cancer: preliminary results-EORTC 22921, I Clin Oncol, 2005;23:5620-7.

14. Collete L, Bosset JF, den Dulk M, et al., Patients with curative resection of CT3-4 rectal cancer after preoperative radiotherapy or radiochemotherapy: does anybody benefit from adjuvant fluorouracil-based chemotherapy? A trial of the European Organisation for Research and Treatment of Cancer Radiation Oncology Group, I Clin Oncol, 2007;25:4379-86.

15. Beets $G$, Maas M, Nelemans P, et al., Evaluation of response after chemoradiation for rectal cancer as a predictive factor for the benefit of adjuvant chemotherapy: a pooled analysis of 2,724 individual patients, J Clin Oncol, 2011;29(Suppl.):Abstract 361

16. Hofheinz R, Wenz F, Post S, et al., Capecitabine (Cape) versus 5-fluorouracil-based (neo)adjuvant chemoradiotherapy for locally advanced rectal cancer: long-term results of a randomized, phase III trial, J Clin Oncol, 2011;29(Suppl.): Abstract 3504.

17. Roh MS, Yothers GA, O'Connell MJ, et al., The impact of capecitabine and oxaliplatin in the preoperative multimodality treatment in patients with carcinoma of the rectum: NSABP R-04, I Clin Oncol, 2011;29(Suppl.): Abstract 3503.

18. Gérard JP, Azria D, Gourgou-Bourgade S, et al., Comparison of two neoadjuvant chemoradiotherapy regimens for locally advanced rectal cancer: results of the phase III trial ACCORD 12/0405-Prodige 2, J Clin Oncol, 2010;28:1638-44.

19. Aschele C, Cionini L, Lonardi S, et al., Primary tumor response to preoperative chemoradiation with or without oxaliplatin in locally advanced rectal cancer: pathologic results of the STAR-01 randomized phase III trial, I Clin Oncol, 2011;29:2773-80.

20. Roedel C, Becker H, Fietkau R, Preoperative chemoradiotherapy and postoperative chemotherapy with 5 -fluorouracil and oxaliplatin versus 5-fluorouracil alone in locally advanced rectal cancer: first results of the German CAO/ARO/AIO-04 randomized phase III trial, Presented at: American Society of Clinical Oncology Annual Meeting, Chicago, US, June 3-7, 2011;Abstract LBA3505.

21. Glynne-Jones R, Grainger J, Harrison J, et al., Neoadjuvant chemotherapy prior to preoperative chemoradiation or radiation in rectal cancer: should we be more cautious? Br J Cancer, 2006;94:363-71.

22. Chau I, Allen M, Cunningham D, et al., Neoadjuvant systemic fluorouracil and mitomycin C prior to synchronous chemoradiation is an effective strategy in locally advanced rectal cancer, Br I Cancer, 2003;88:1017-24.

23. Chau I, Brown G, Cunningham D, et al., Neoadjuvant capecitabine and oxaliplatin followed by synchronous chemoradiation and total mesorectal excision in magnetic resonance imaging-defined poor-risk rectal cancer, I Clin Oncol, 2006;24:668-74.

24. Chua YJ, Barbachano Y, Cunningham D, et al., Neoadjuvant capecitabine and oxaliplatin before chemoradiotherapy and total mesorectal excision in MRI-defined poor-risk rectal cancer: a phase 2 trial, Lancet Oncol, 2010;11:241-8.

25. Calvo FA, Serrano FJ, Diaz-Gonzalez JA, et al., Improved incidence of pTO downstaged surgical specimens in locally advanced rectal cancer (LARC) treated with induction oxaliplatin plus 5-fluorouracil and preoperative chemoradiation Ann Oncol, 2006;17:1103-10.

26. Koeberle $D$, Burkhard $R$, von Moos $R$, et al., Phase II study of capecitabine and oxaliplatin given prior to and concurrently with preoperative pelvic radiotherapy in patients with locally advanced rectal cancer, Br J Cancer, 2008;98:1204-9.

27. Gunnlaugsson $A$, Anderson $H$, Fernebro E, et al., Multicentre phase II trial of capecitabine and oxaliplatin in combination with radiotherapy for unresectable colorectal cancer: the CORGI-L Study, Eur I Cancer , 2009:45:807-13.

28. Fernández-Martos C, Pericay C, Aparicio J, et al., Phase II, randomized study of concomitant chemoradiotherapy followed by surgery and adjuvant capecitabine plus oxaliplatin (CAPOX) compared with induction CAPOX followed by concomitant chemoradiotherapy and surgery in magnetic resonance imaging-defined, locally advanced rectal cancer: Grupo cancer de recto 3 study, J Clin Oncol, 2010;28:859-65.

29. Fernandez-Martos C, Pericay C, Salud A, et al., Three-year outcome of GCR-3: a phase II randomized trial comparing conventional preoperative chemoradiation (CRT) followed by surgery and postoperative adjuvant chemotherapy (CT) with induction CT followed by CRT and surgery in locally advanced rectal cancer, I Clin Oncol, 2011:29(Suppl):Abstract 3552.

30. Marechal R, Vos B, Polus M, et al., Short course chemotherapy followed by concomitant chemoradiotherapy and surgery in locally advanced rectal cancer: a randomized multicentric phase II study, Ann Oncol, 2011; Oct 29 [Epub ahead of print].

31. Rödel C, Arnold D, Becker H, et al., Induction chemotherapy before chemoradiotherapy and surgery for locally advanced rectal cancer : is it time for a randomized phase III trial? Strahlenther Onkol, 2010;186:658-64. 